\title{
Nasal Cavity and Paranasal Sinus Cancer by AJCC v6 Stage
}

National Cancer Institute

\section{Source}

National Cancer Institute. Nasal Cavity and Paranasal Sinus Cancer by A/CC v6 Stage. NCI

Thesaurus. Code C90528.

A term that refers to the staging of nasal cavity and paranasal sinus carcinoma according to the American Joint Committee on Cancer, 6th edition. 